\title{
2-Dimensional Mapping of Damage in Moisture Contaminated Polymer Composites Using Dielectric Properties
}

\section{OGHENEOVO IDOLOR ${ }^{1}$, RISHABH GUHA ${ }^{1}$, LOGAN BILICH ${ }^{1}$ and LANDON GRACE ${ }^{1}$}

\begin{abstract}
Polymer composite materials are increasingly being adopted in civil infrastructure, oil \& gas, marine, automotive and aerospace industries. Achieving ubiquitous adoption would require new advances in development of non-destructive examination methods which are cost effective relative to composite structures under test, simple to utilize and sensitive enough to ascertain the structural integrity of composite parts, especially in the absence of visible damage. This study proposes a relatively simple method for non-destructive testing of polymer composites that detects 2-dimensional micro to nano-scale damage by analysis of the effects of polymer-moisture interactions on microwave-frequency dielectric properties. In this study, we apply this method to mapping of internal damage in two widely used aerospace composite laminates; a 12-ply bismaleimide/quartz and a 16-ply epoxy/7781 fiber glass composite laminate. Specimens are dried and then immersed in deionized water, to simulate long term exposure to humid environments while measuring gravimetric moisture uptake. Localized damage is induced in the laminate specimens via low velocity impact damage of 5 and 9 Joules. A split post dielectric resonator coupled with a vector network analyzer is used to determine the spatial variation in relative permittivity across the composite laminate. Generally, results show significant increase in relative permittivity towards the center of impact damage compared to surrounding undamaged areas. This increase is indicative of internal damage as a result of micro-crack formation around the point of impact. This new free volume in the damaged area is primarily occupied by free water; driving a local increase in the relative permittivity in the damaged area due to the locally-higher ratio of free to bound water. Due to the tendency of polymer composites to absorb measurable absorbed moisture in almost all environments, this relatively simple non-destructive examination method using water as a type of "imaging agent" demonstrates considerable promise for early detection of damage.
\end{abstract}

${ }^{1}$ North Carolina State University, Raleigh, NC 27695 


\section{INTRODUCTION}

Polymer matrix composites are increasingly substituted for traditional materials in infrastructure, oil and gas, marine and automotive industries due to their numerous and well-known advantages [1,2]. This increase in adoption has driven the need for development of bespoke non-destructive examination (NDE) methods for composite materials which are reliable, cost effective, and easy to use. Various advances have been made in developing NDE techniques for composites. These techniques are designed to detect hot spots within the material such as delaminations, voids, defects, inclusions, and porosity. These include generally applied NDE techniques like ultrasound and X-ray CT, as well as more tailored approaches such as thermography, shearography, and microwaves. Application of the various techniques are limited by the part geometry, material type, thickness, size of defect, and depth of defect[3,4]. The microwave technique has grown in popularity even with limitations to nonconductive polymer composites due to its relatively low capital investment and simplicity. These techniques involve the use of low power electromagnetic signals (approx. $1-40 \mathrm{~mW}$ ) in the microwave frequency range. The microwave technique can be applied in several ways, one of which involves the use of a resonant cavity. This approach involves determining the dielectric properties at a fixed frequency and tracking changes resulting from internal defects and material damage. By monitoring the resonant frequency and its quality factor within this cavity, dielectric properties can be calculated [5]. The resonant frequency is highly sensitive to temperature, humidity, and the nature and volume of foreign bodies introduced into the resonant cavity[6]. Shifts in resonant frequency present a way to detect damage within a moisture contaminated polymer composite with relatively limited sensitivity. This study proposes a way to enhance the sensitivity of the resonant cavity method for NDE of non-conductive polymer composites by exploiting the dynamic behavior of moisture within a damaged composite laminate.

Polymer composites have a tendency to absorb measurable mounts of moisture in nearly all environments [7]. It has been well-reported that this affects mechanical, chemical, and dielectric properties [8,9]. Microwave frequency dielectric properties are very sensitive to moisture due to the dipolar nature of the water molecule. At these frequencies, the dipolar rotational polarization mechanism becomes active in an applied electromagnetic field, and dominates contribution to dielectric properties compared to other active atomic and electronic polarization mechanisms [10]. Depending on its state, absorbed moisture responds to an applied electromagnetic field in different ways. When in the 'free' state, without any secondary bonding interaction with the polymer network, water molecules rotate with a changing electromagnetic field without any restriction. In the 'bound' state, water molecules are subject to interactions with the polymer network that impose various degrees of restriction to its dipolar rotation [11].

The ability of a water molecule to rotate in an applied electromagnetic field is closely related to its dipole moment, which is closely related to the real part of relative permittivity (RP). Free water without restriction to rotation has a relatively high relative permittivity $(\sim 80)$ compared to bound water [12-14]. Bound water has significantly lower RP that varies according to the nature of the imposed restriction to rotation [9]. These restrictions range from weakly interacting van der Waals forces and dipole-dipole attractions to highly-restrictive hydrogen bonding [11]. The bulk RP of a 
polymer composite material consist of contributions from the polymer matrix, reinforcing fibers, bound water and free water. All of these possess RPs which are relatively low except for free water having a significantly higher RP. Hence, very small quantities of absorbed moisture in the free state within the composite material would cause a measurable increase in relative permittivity.

In previous work, we have reported on RP indications of damage within a polymer matrix, the effect of damage on the free and bound water interactions with the polymer matrix, and how this relates to changes in the bulk relative permittivity [6]. In this work, we extend this method to mapping of localized damage which exists within a composite laminate. Using a split post dielectric resonator (SPDR) operating at $2.45 \mathrm{GHz}$, the spatial variation in bulk RP over the composite laminate can be obtained and then used to predict location and extent of damage within a composite laminate.

\section{MATERIALS AND METHODS}

\section{Materials}

A bismaleimide (BMI)/quartz fiber laminate representative of a typical aerospace radome composite was tested. The matrix is a bismaleimide resin, trade name HexPly® F650. The reinforcing fibers are quartz woven into an 8-harness satin fabric. A 12-ply test panel fabricated with dimensions of $36 \times 36$ inches was cured in an autoclave at $190^{\circ} \mathrm{C}$ and 85 psi for 4 hours. This was followed by an 8-hour post-cure at $232^{\circ} \mathrm{C}$ according to manufacturer's recommendations. Individual $132 \times 75 \mathrm{~mm}$ test specimens were cut from the larger panel. The laminate properties were obtained by means of resin burn-off in a high-temperature furnace maintained at $800^{\circ} \mathrm{C}$ until all bismaleimide residue was eliminated. Pre- and post-burn weight measurements allowed the accurate determination of resin, void, and fiber content. In order to ensure accuracy, 15 samples from various locations within the 36-inch square panel were used. Each sample weighed approximately 5 grams prior to burn off. The average laminate thickness was $2.6 \mathrm{~mm}$. Other laminate properties are given in Table I.

An epoxy/glass fiber laminate typically used in aerospace service was similarly used as test specimens. The matrix is an epoxy resin, trade name Hexcel F161. The reinforcement was an 8-harness satin weave glass fabric, style 7781.16 plies of prepreg with dimensions $12 \times 7$ inches were laid up to form a flat laminate. This was cured in a hot press at 56 psi. The prepreg laminate was warmed up from room temperature at an average rate of $10^{\circ} \mathrm{C} / \mathrm{min}$ to $180^{\circ} \mathrm{C}$. The laminate was held at $180^{\circ} \mathrm{C}$ for 2 hours, pressure was then released and the laminate was cooled at an average rate of $2^{\circ} \mathrm{C} / \mathrm{min}$ to room temperature. Individual $132 \times 60 \mathrm{~mm}$ test specimens were cut from the larger panel. Laminate properties were obtained by means of resin burn-off in a high-

TABLE I. AVERAGE BMI/QUARTZ FIBER LAMINATE PROPERTIES.

\begin{tabular}{|l|c|c|}
\hline \multicolumn{1}{|c|}{ Property } & $\begin{array}{c}\text { Mean } \\
(\mathbf{\%})\end{array}$ & $\begin{array}{c}\text { Standard Deviation } \\
(\mathbf{\%})\end{array}$ \\
\hline Fiber volume fraction & 59.41 & 0.149 \\
\hline Resin volume fraction & 40.27 & 0.144 \\
\hline Voids & 0.32 & 0.045 \\
\hline
\end{tabular}


TABLE II. AVERAGE EPOXY/GLASS FIBER LAMINATE PROPERTIES.

\begin{tabular}{|l|c|c|}
\hline \multicolumn{1}{|c|}{ Property } & $\begin{array}{c}\text { Mean } \\
(\mathbf{\%})\end{array}$ & $\begin{array}{c}\text { Standard Deviation } \\
(\mathbf{\%})\end{array}$ \\
\hline Fiber volume fraction & 62.25 & 0.105 \\
\hline Resin volume fraction & 31.95 & 0.228 \\
\hline Voids & 5.80 & 0.215 \\
\hline
\end{tabular}

temperature furnace maintained at $800^{\circ} \mathrm{C}$ until elimination of all epoxy residue. Preand post-burn weight measurements allowed the accurate determination of resin, void, and fiber content. The average laminate thickness was $2.7 \mathrm{~mm}$. Other laminate properties are given in Table II.

Specimens were cut into test sizes using a wet diamond saw. These were then dried in a vacuum oven at $65^{\circ} \mathrm{C}$ until a stable weight was achieved according to ASTM D5229. Using a water bath set at a fixed temperature of $25^{\circ} \mathrm{C}$, the epoxy/glass fiber and BMI/quartz test specimens were immersed in de-ionized water. Gravimetric moisture uptake was measured using a high precision analytical balance.

Impact damage - at a level assumed to be difficult to discern with coating/paint applied - was induced in the test specimens. For both material types low velocity hail impact damage was simulated using a drop tower equipped with a hemispherical impactor tip. By varying the drop height, the 16-ply epoxy/glass fiber specimens were impacted at 5 joules. While the 12-ply BMI/quartz specimens were impacted at 9 joules. Images of impacted specimen and typical specimen size are shown in Figure 1.

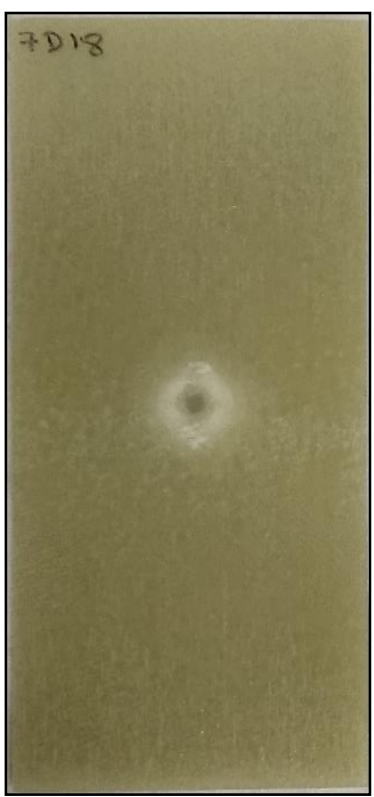

(a)

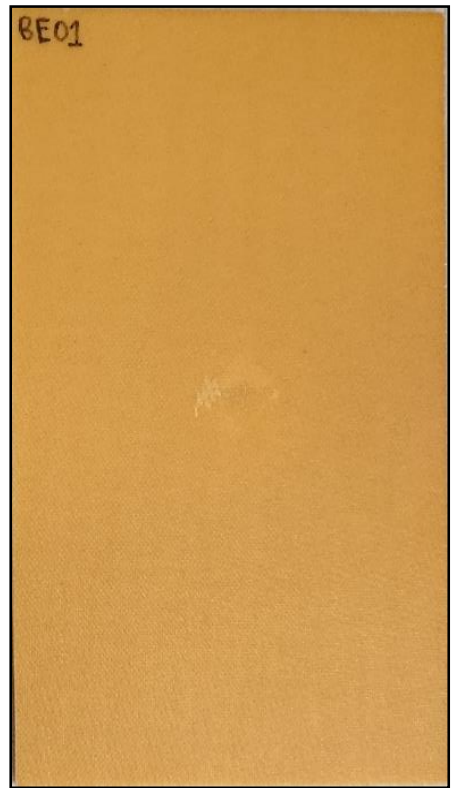

(b)

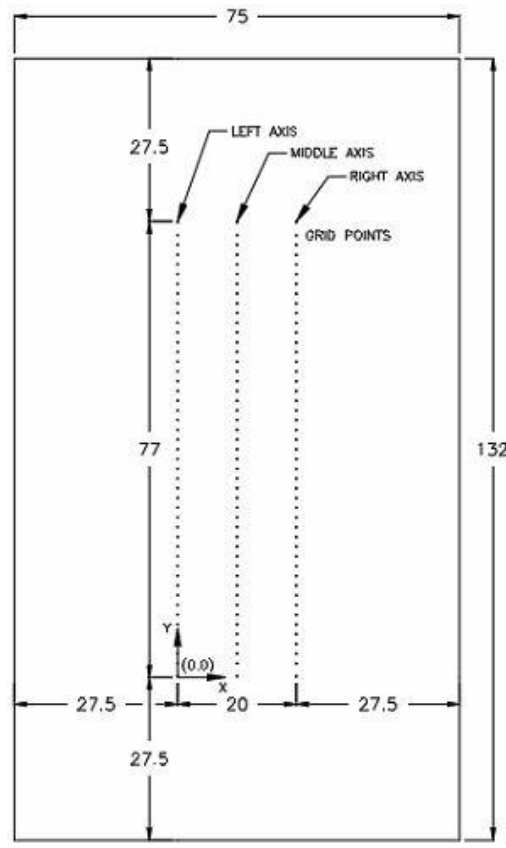

(c)

Figure 1. (a) BMI/quartz specimen showing visible damage due to $9 \mathrm{~J}$ impact (b) Epoxy/glass fiber specimen showing visible damage due to $5 \mathrm{~J}$ impact (c) Layout of measurement grid points on a typical BMI/quartz specimen (dimensions in $\mathrm{mm}$ ). 


\section{Experimental Setup}

To accurately measure dielectric properties at microwave frequencies, a split post dielectric resonator (SPDR) fabricated by QWEDC of Warsaw, Poland was coupled with an Agilent programmable vector network analyzer (VNA) as shown in Figure 2. This setup enables measurement of bulk relative permittivity and tracking of small changes on the order of $10^{-3}$. Measurement uncertainty using this setup is as low as $0.3 \%$ in relative permittivity, and loss tangent can be measured with resolutions as low as $2 \times 10^{-5}$ [15]. Additional details on the calibration and setup of the system has been reported in our previous work [6]. On insertion of the specimen under test into the device, the resonant frequency undergoes a measurable shift, measured using the VNA. This resonant frequency shift is used to calculate the real part of relative permittivity $\left(\varepsilon_{r}^{\prime}\right)$ of the specimen according to equation 1 .

$$
\varepsilon^{\prime}{ }_{r}=1+\frac{f_{0}-f_{s}}{h f_{0} K_{\varepsilon}\left(\varepsilon^{\prime}{ }_{r}, h\right)}
$$

where

$f_{0} \quad$ is resonant frequency of empty SPDR

$f_{s} \quad$ is resonant frequency with the dielectric specimen inserted

$h$ is sample thickness

$K_{\varepsilon} \quad$ is a function of $\varepsilon^{\prime}{ }_{r}$ and $h$, documented in a table unique to each SPDR and provided by the manufacturer

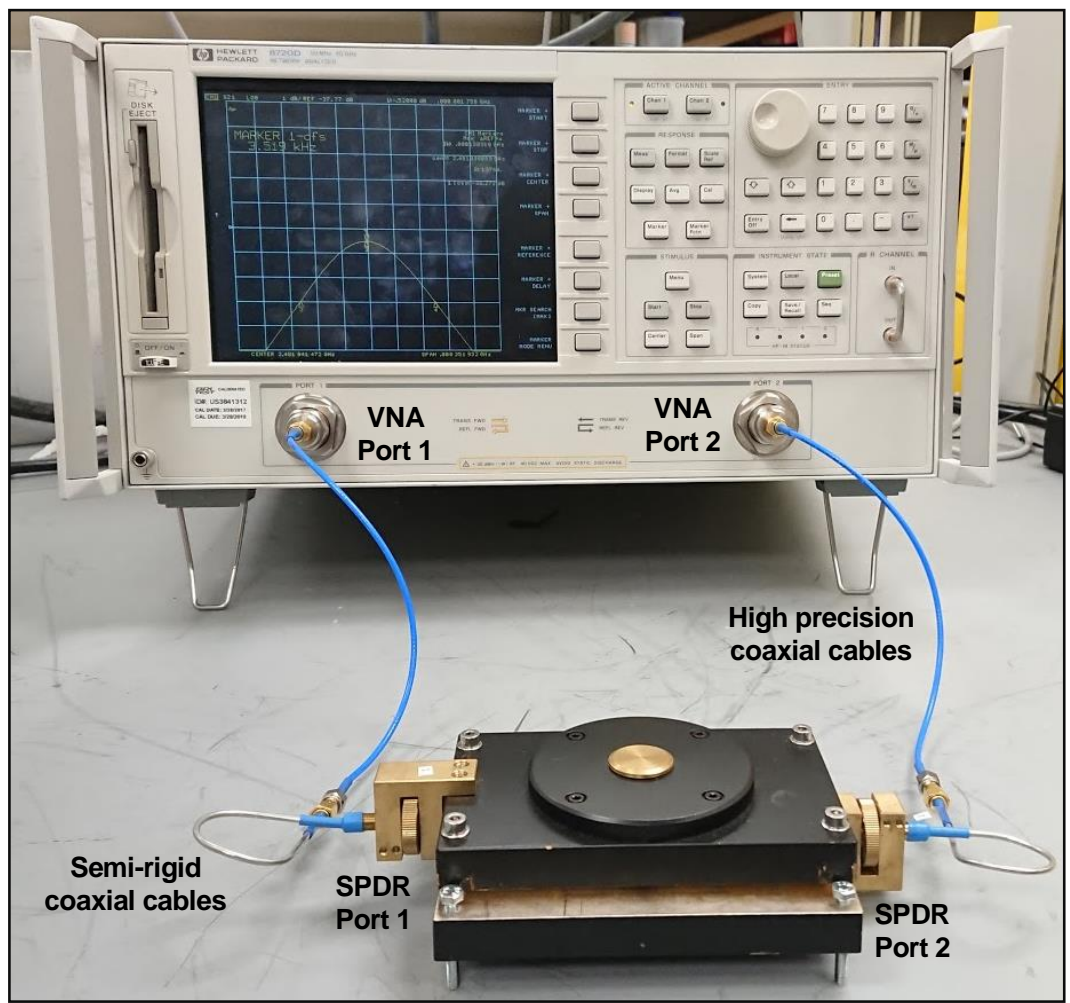

Figure 2. Split post dielectric resonator (SPDR) coupled to Vector Network Analyzer (VNA). 
Weight and spatial RP at $2.45 \mathrm{GHz}$ were measured at specific intervals within the immersion period of test specimens. For each measurement, specimens were first carefully dried using a lint-free cloth. To eliminate the effects of residual surface moisture, specimens were allowed to dry further at room temperature for approximately 10 minutes to achieve a stable weight. To ensure accuracy, static electric charges on the specimen surface were eliminated by exposure to air containing positively and negatively charged ions supplied from an ionizing gun immediately prior to weighing. Specimen weights were recoded before and after each 25 minute test and an average weight was used to calculate the percentage moisture concentration for that measurement.

To accurately move the specimen in the SPDR, a setup employing NEMA 17 stepper motors controlled by an Arduino MEGA 2560 board was utilized. MATLAB scripts were developed to simultaneously control the VNA and accurately move the sample to the measurement grid points. Depending on the sample size and spatial area to be covered, a surface grid of appropriate dimension was created with a step size of $0.5 \mathrm{~mm}$. To eliminate specimen edge effects, the grid layout ensured the grid points at the margins were at least $27.5 \mathrm{~mm}$ from any edge of the specimen (see Figure 1(c)). On each step, 3 measurements of the local bulk relative permittivity for an approximately $55 \mathrm{~mm}$ diameter circular area (the dimensions of the resonant cavity itself) were taken. An average RP was then calculated and assigned to the grid point at the center of the resonator.

\section{RESULTS AND DISCUSSIONS}

The resonant frequency is sensitive to changes in specimen thickness, sample moisture content, as well as temperature and humidity of air within the resonant cavity. The effect of these on the data obtained had to be eliminated or compensated for if significant. In our previous work, we have reported on the effect of temperature and humidity changes on the resonant frequency of the empty resonator and how this affects the accuracy of the SPDR method [6]. Due to the extended duration of the test, temperature and humidity of the test environment were controlled to ensure fluctuation were kept below levels significant enough to affect RP changes due to damage. To eliminate the effect of moisture desorbed or absorbed by the test specimen during test, differences in specimen weight before and after the test were determined and RP adjusted accordingly based on the average change in RP per percent change in moisture content measured for each material type. The effects of specimen thickness variation were eliminated by first collecting data on an undamaged dry sample before inducing impact damage. The RP obtained for each grid point was then subtracted from the RP of similar grid points obtained for other conditions which were subsequently measured. Such conditions include specimen impacted but dry and other conditions where the specimen was moisture contaminated after impact having varying levels of moisture content. To clearly demonstrate the effectiveness of this approach, the effect of moisture uptake alone on the RP across the specimen was also investigated. Figure 3 shows the variation in RP across the middle grid points of an undamaged $\mathrm{BMI} /$ quartz specimen. The $0 \%$ moisture curve shows the RP variation for a dry undamaged sample, while the $0.1 \%$ moisture curve shows variation along the 
same axis for the same specimen now containing $0.1 \%$ moisture. The 'thickness' curve, plotted with reference to the right

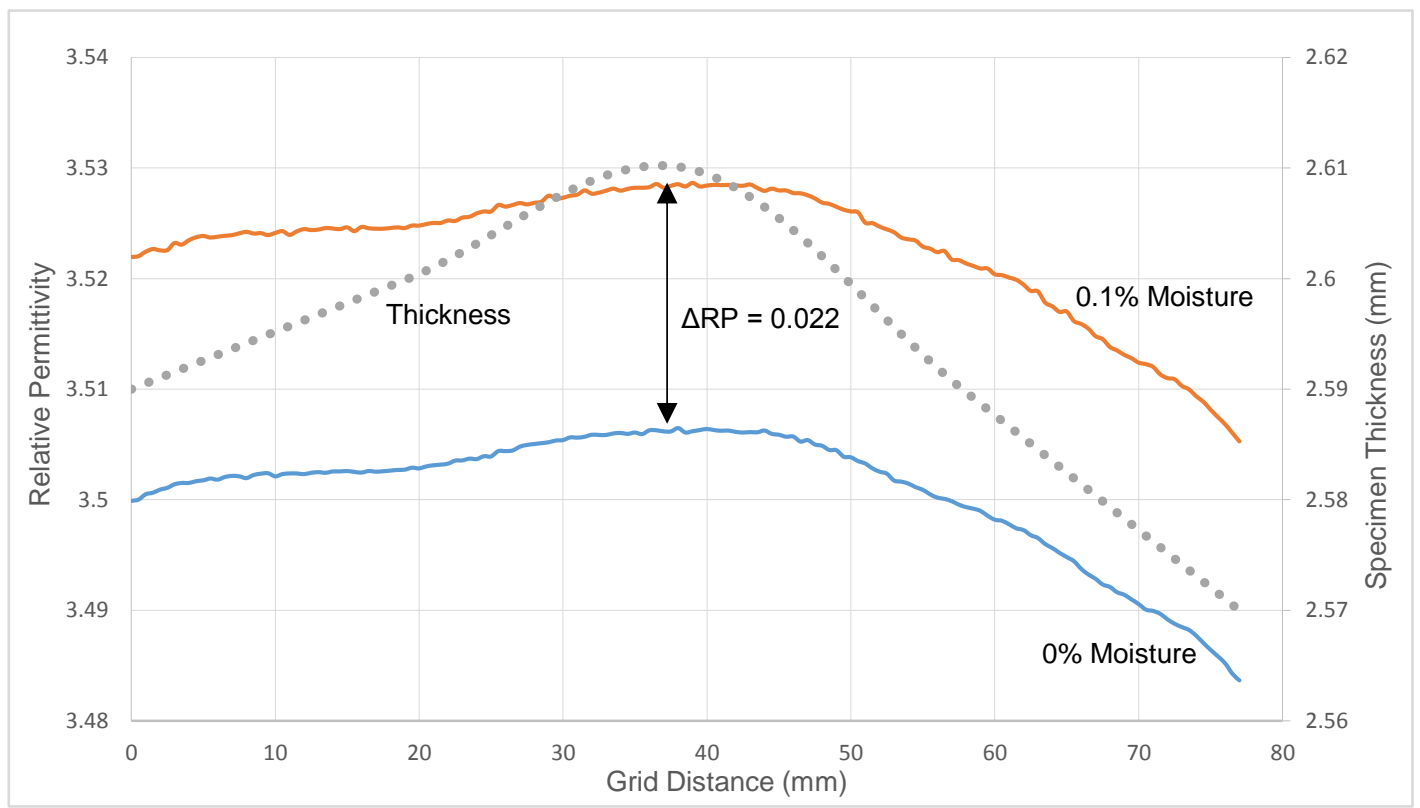

Figure 3. Relative Permittivity (RP) variation along the middle axis of a BMI/Quartz specimen.

ordinate axis, shows the slight variations in thickness across the specimen (2.57$2.61 \mathrm{~mm}$ ). The initial variation in the RP across this specimen can be attributed to change in volume effects such as thickness and porosity across the length of the specimen. In this case, the variation due to thickness dominates when compared to other non-uniformities, evident from the clear correlation between RP variation and thickness for both the dry and moisture contaminated states of the specimen. According to Figure 3, the presence of $0.1 \%$ moisture in the undamaged specimen causes a uniform rise of approximately 0.022 in RP across the specimen. This variation is shown to be consistent across the length of the specimen. This observation suggests, by subtracting the initial dry RP without impact damage, the effects of volume changes (thickness variation) are eliminated from the results.

With the effects of specimen thickness changes and moisture contamination on RP clearly understood and isolated, the effect of moisture contamination on the RP of specimens damaged by impact could then be investigated. Note that during RP measurements of the dry specimens, some moisture is absorbed from the environment, which gives an average moisture content of slightly above zero but generally less than $0.03 \%$. This is assumed to be $0 \%$ in this study.

Figure 4 shows the spatial variation in RP over the surface of an impacted and moisture contaminated BMI/quartz specimen. RP measurements are taken at each grid point along the three axis of grid points (left, middle and right axis), see grid point layout in Figure 1(c). The middle axis shows higher increase in RP at the damaged region when compared with the left and right axis. Focus would be on analyzing the variations in RP along this axis only, as other axis would show similar but less significant changes in RP resulting from damage, clearly evident in figure 4.

Figure 5(a) and (b) show the variations of RP on grid points on the middle axis along the length of the specimen after subtraction of the dry undamaged RP, at levels 
of moisture contamination ranging from $0 \%$ to $0.7 \%$. At $0 \%$ moisture after impact, a slight depression in RP is observed around the impact location. This can be attributed to the slight depression created by the impactor at the impact point on the specimen

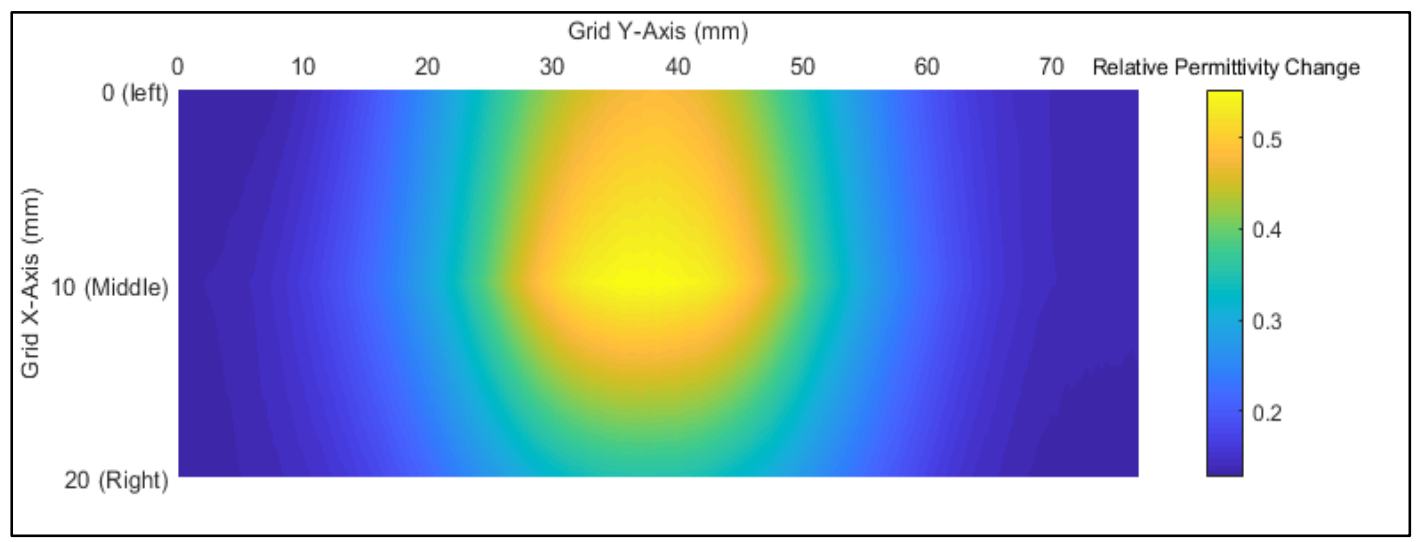

Figure 4. Change in RP across the left, middle and right axis grid points (See fig 1(c) for grid layout) for a BMI/Quartz specimen impacted at $9 \mathrm{~J}$ with $0.7 \%$ moisture content.

surface and damage within the composite laminate as a result of the impact. This change in RP represents the sensitivity of the resonant cavity method to material volume changes alone.

Considering the moisture-contaminated conditions, for the BMI/quartz specimen the RP at grid points less than $5 \mathrm{~mm}$ from the end grid points of the specimen is observed to be relatively constant at each moisture level (grid start and end points to point 'A', see Figure 5(a)). While for the epoxy/glass fiber with less damage, this is observed below 10mm (grid start and end points to point 'A', see Figure 5(b)). This can be attributed to those being undamaged regions which uptake moisture at similar rate. For grid points within A-A; a gradual increase in RP is observed from the edges towards the impacted point at the center, increasing to a maximum at the impact location. As the moisture level increases, this behavior is observed to generally remain consistent, but steeper increases in RP is observed towards the impact location as moisture levels increase. This behavior is consistent with the effects of moisture in the free and bound states $[9,16]$. Impact-induced localized delamination and micro-cracks at the damaged region serve as micro-voids in the polymer matrix. Water molecules which have a preference for the 'free state' compared to the 'bound state'[17], preferentially migrate to these micro-voids forming clusters of free water. This free water, which exhibits a higher RP compared to other contributors, causes a significant increase in the local bulk RP.

Applying this methodology to NDE of polymer composites involves determining the location and size of the visible and, more importantly, non-visible (internal) damaged regions. By observing the response of the SPDR, it was noted that changes in resonant frequency is such that a disturbance or foreign body closer to the edge of the cavity causes less of a shift compared to those in the center, which cause the largest change in resonant frequency. This explains the dumbbell shapes of the RP variation across specimens damaged at the center. By testing the $2.45 \mathrm{GHz}$ SPDR, it was observed that any disturbance or foreign body within $22 \mathrm{~mm}$ radius from the center of the resonant cavity created a resonant frequency shift. With this information 
and knowing the grid point at which the damaged region begins causing an increase in RP (lines labeled ' $A$ ' in Figure 5(a) and (b)), the location and extent of internal damage can be estimated. The center of the damaged region is simply the point with maximum RP, consistent with the locally-highest concentration of free water as a result of the greatest level of damage.

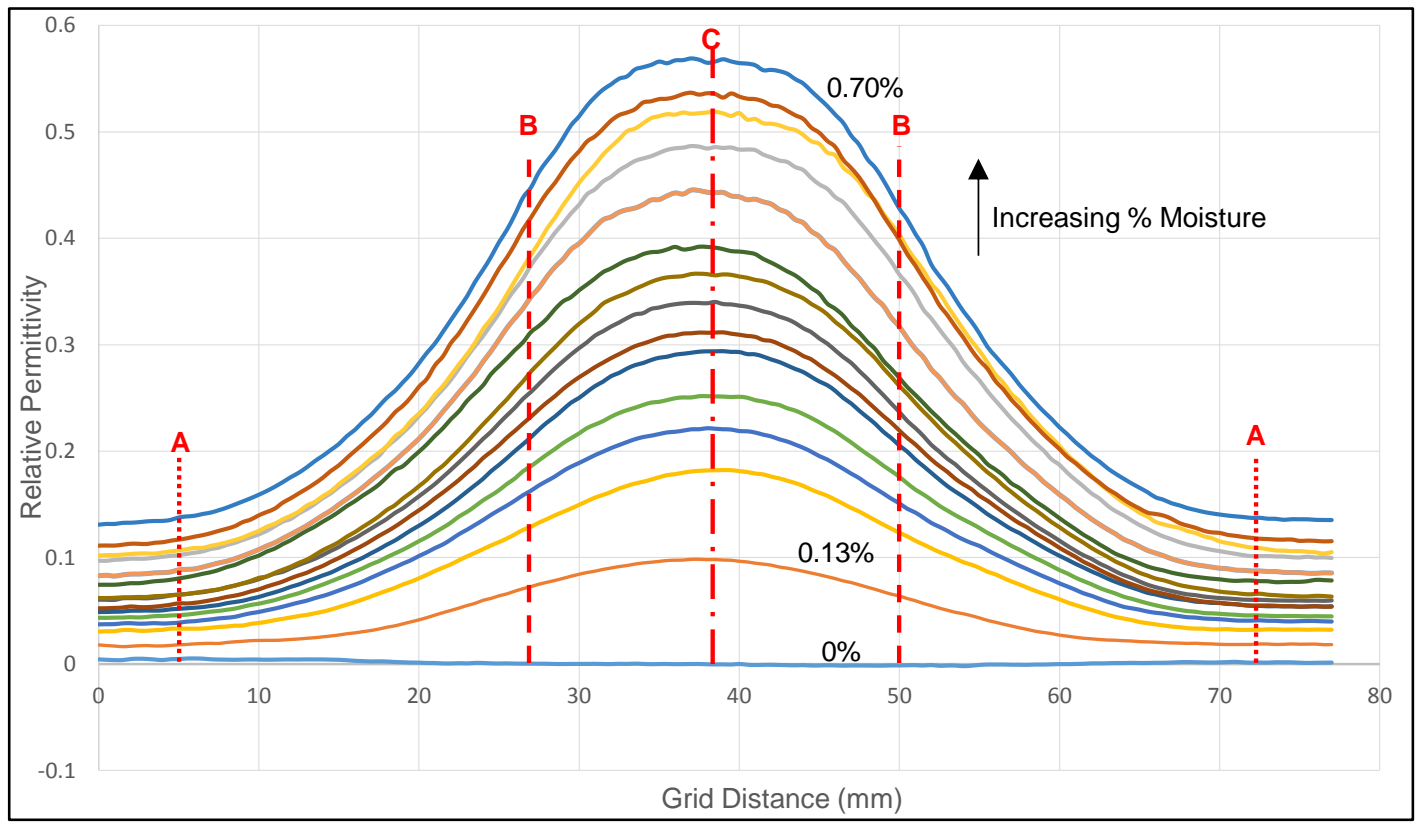

(a)

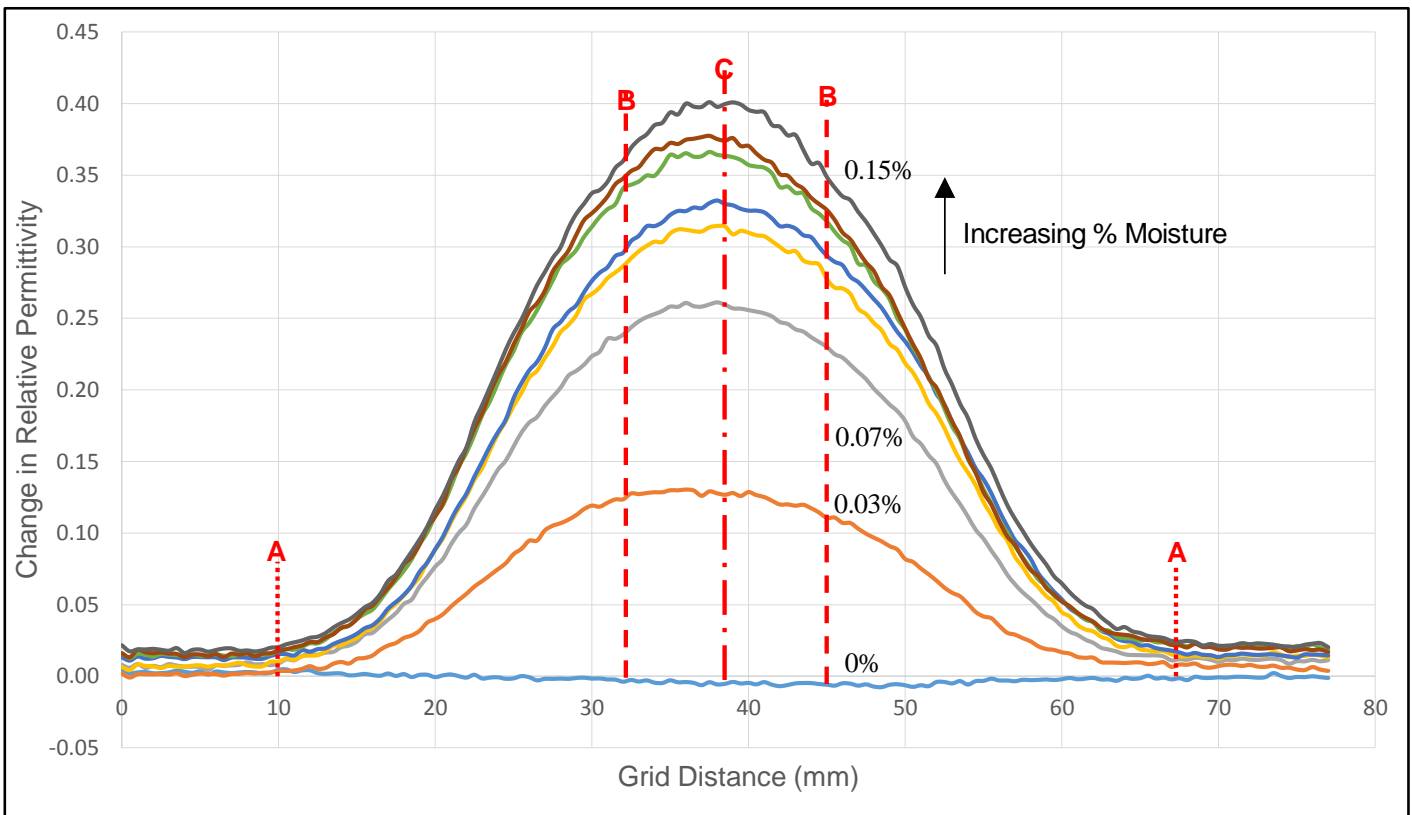

(b)

Point A - where the damaged region begin to cause frequency shifts

Point $\mathrm{B}$ - limits of the damaged region

Point $\mathrm{C}-$ Center of the damaged region

Figure 5. Change in RP along the middle axis for center impacted (a) BMI/quartz specimen impacted at $9 \mathrm{~J}$ (b) Epoxy/glass fiber specimen impacted at $5 \mathrm{~J}$. 
The center and extent of damage are shown for the BMI/quartz and epoxy/glass fiber specimens in Figure 5(a) and (b) respectively. The results generally agree with visual examination of laminate specimen damaged regions when observed under a light source. Further studies are required to determine resolution and application limits of the method. Also, understanding the effects of moisture content level on these requires further investigation.

Finally, to realistically represent an in-service composite which experiences long term moisture contamination before damage; a dry undamaged specimen was moisture contaminated to the approximate equilibrium content for a composite exposed to room conditions, before inducing damage via impact at 9 joules. The average equilibrium moisture content of undamaged $\mathrm{BMI} / \mathrm{quartz}$ laminate at room conditions (approximately $25^{\circ} \mathrm{C}$ and $55-65 \%$ humidity) was estimated to be between $0.2-0.3 \%$. Curves A-E in Figure 6 show the uniform rise in RP across the middle axis of the specimen, as the specimen becomes moisture contaminated up to equilibrium levels. Curve $\mathrm{F}$ shows the variation in RP immediately after impact and at similar moisture content as E. A slight depression around the impact point is observed in curve F, when compared to $\mathrm{E}$. This can be attributed to slight surface variation and internal fractures on impact, comparable to similar depression in the previously discussed dry specimens. At approximately 1, 2, 7 and 11 days in similar environmental conditions, curves G, H, I and J are recorded. During this period an increase of $0.06 \%$ in moisture content is observed. These show a gradual increase in RP around the damaged region compared to the curve F (immediately after impact). This is indicative of free water gradually migrating to micro-cracks within the damaged region, similar to the case with the dry specimen previously discussed. However, in this case there is uncertainty if the moisture migrating to these micro-cracks are: newly absorbed molecules preferentially migrating to the micro-cracks to exist in the free state, or water molecules previously bound to the polymer matrix migrating to these micro-cracks, or a combination of both scenarios. Previous studies have shown a preference for water to exist in the free state, with higher tendencies to form hydrogen bonds with other water molecules compared to forming bonds with the polymer network [17]. 


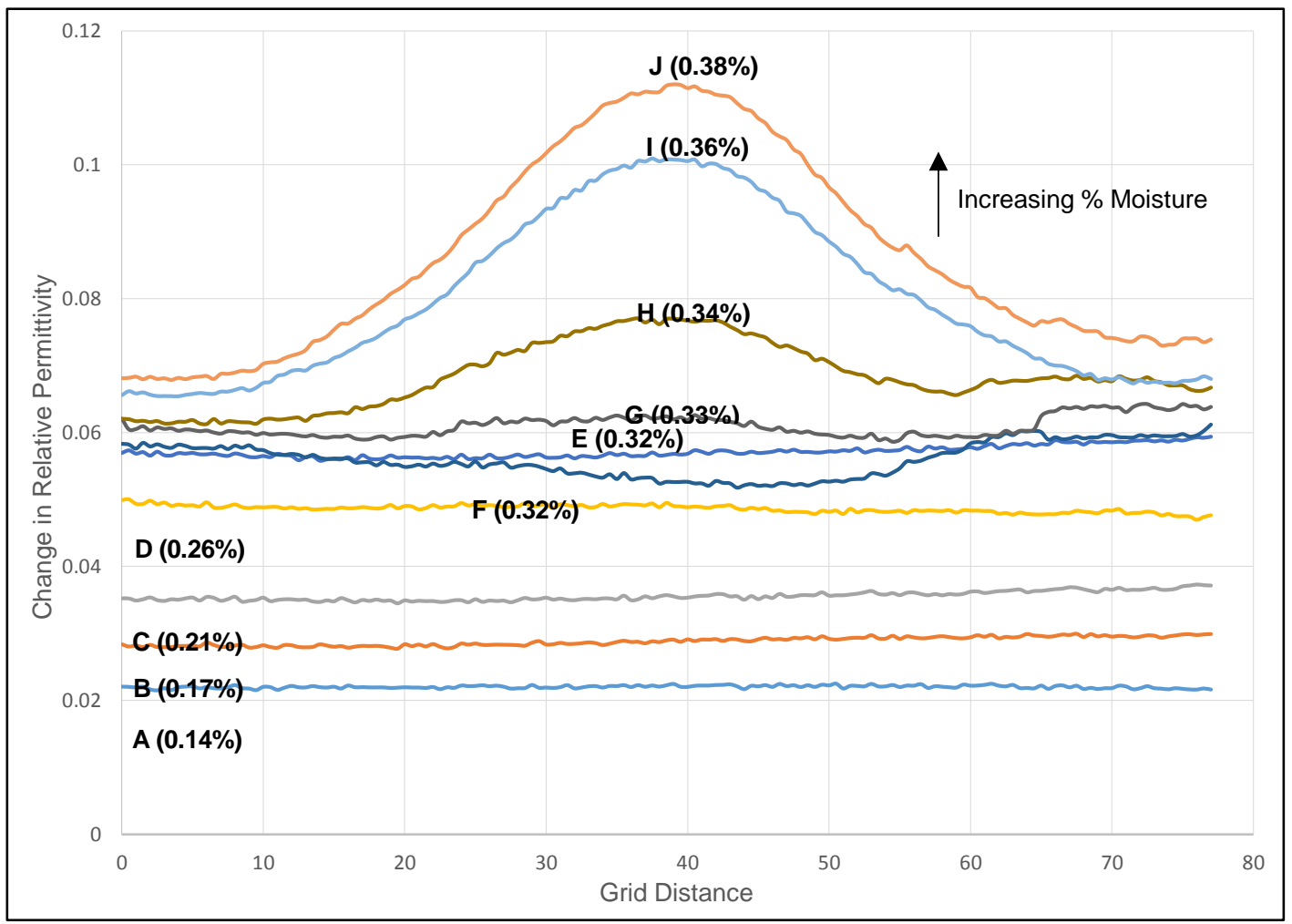

Figure 6. Change in RP along the middle axis for center impacted quartz/BMI specimen impacted at $9 \mathrm{~J}$ after moisture contamination, values in parentheses represent $\%$ moisture for each curve.

\section{CONCLUSION}

Typical aerospace polymer composites were impacted before and after moisture contamination inducing low levels of localized damage within the laminates. A resonant cavity technique employing a split post dielectric resonator (SPDR) coupled with a vector network analyzer (VNA) was employed to determine the relative permittivity (RP) across the surface of damaged and undamaged composite laminates. Temperature and humidity variations were controlled to minimize their effects on RP variation during the test; while, thickness and moisture content variation effects on RP were compensated for. RP data obtained across the damaged laminate was analyzed to determine the location and extent of damage within the composite laminate. The location (center) of the damaged region was determined as the point with maximum $\mathrm{RP}$, while the extent of localized damage was determined from the point where the resonant frequency of the resonant cavity begins to shift due to the damaged region. Results obtained were generally consistent across BMI/quartz and epoxy/fiber glass specimens, and were in agreement with results from visual inspection of damaged regions when viewed in front of a bright light source. The study demonstrated that applying the dynamic behavior of moisture to damage characterization in polymer composites can significantly enhance the sensitivity of resonant cavity microwave 
methods by as much as 40 times. However, this is strongly dependent on the initial moisture content of the composite, magnitude of damage, and extent of moisture exposure. These preliminary studies indicate damage is detectable by this method at moisture concentrations as low as $0.03 \%$ by weight. Further studies will determine the resolution limits and application boundaries of the method.

\section{ACKNOWLEDGMENTS}

This material is based upon work partially supported by the National Science Foundation under Grant No. CMMI-175482.

\section{REFERENCES}

[1] G. Neşer, Polymer based composites in marine use: History and future trends, Procedia Eng. 194 (2017) 19-24. doi:10.1016/j.proeng.2017.08.111.

[2] A.E. Krauklis, A.I. Gagani, A.T. Echtermeyer, Near-infrared spectroscopic method for monitoring water content in epoxy resins and fiber-reinforced composites, Materials (Basel). 11 (2018). doi:10.3390/ma11040586.

[3] P. Duchene, S. Chaki, A. Ayadi, P. Krawczak, A review of non-destructive techniques used for mechanical damage assessment in polymer composites, J. Mater. Sci. 53 (2018) 7915-7938. doi:10.1007/s10853-018-2045-6.

[4] V. Dattoma, F. Panella, A. Pirinu, A. Saponaro, Advanced NDT Methods and Data Processing on Industrial CFRP Components, Appl. Sci. 9 (2019) 393. doi:10.3390/app9030393.

[5] J. Krupka, Precise measurements of the complex permittivity of dielectric materials at microwave frequencies, Mater. Chem. Phys. 79 (2003) 195-198. doi:10.1016/S02540584(02)00257-2.

[6] O. Idolor, R. Guha, L. Grace, A dielectric resonant cavity method for monitoring of damage progression in moisture-contaminated composites, in: 33rd Tech. Conf. Am. Soc. Compos. 2018, 2018.

[7] C. Hammond, J. Carroll, Environmental effects on composites, in: 19th Struct. Struct. Dyn. Mater. Conf. Am. Inst. Aeronaut. Astronaut. Bethesda, Maryland., 19th Structures, Structural Dynamics and Materials Conference, American Institute of Aeronautics and Astronautics, Bethesda, Maryland, Bethesda, Maryland, 1978: pp. 78-498.

[8] P. Musto, G. Ragosta, L. Mascia, Vibrational spectroscopy evidence for the dual nature of water sorbed into epoxy resins, Chem. Mater. 12 (2000) 1331-1341. doi:10.1021/cm9906809.

[9] L.R. Grace, The effect of moisture contamination on the relative permittivity of polymeric composite radar-protecting structures at X-band, Compos. Struct. 128 (2015) 305-312. doi:10.1016/j.compstruct.2015.03.070.

[10] G. Giannoukos, M. Min, T. Rang, Relative complex permittivity and its dependence on frequency, World J. Eng. 14 (2017) 532-537. doi:10.1108/WJE-01-2017-0007.

[11] Y. Takeshita, E. Becker, S. Sakata, T. Miwa, T. Sawada, States of water absorbed in waterborne urethane/epoxy coatings, Polym. (United Kingdom). 55 (2014) 2505-2513. doi:10.1016/j.polymer.2014.03.027.

[12] D.P. Fernández, Y. Mulev, A.R.H. Goodwin, J.M.H.L. Sengers, A Database for the Static Dielectric Constant of Water and Steam, J. Phys. Chem. Ref. Data. 24 (1995) 33-70. doi:10.1063/1.555977.

[13] V. Komarov, S. Wang, J. Tang, Permittivity and Measurements, Encycl. RF Microw. Eng. (2005). doi:10.1002/0471654507.eme308.

[14] M.T. Hallikainen, F.T. Ulaby, M.C. Dobson, M.A. El-Rayes, L.-K. Wu, Microwave Dielectric Behavior of Wet Soil - Part I: Emprical Models and Experimental Observations., IEEE Trans. Geosci. Remote Sens. GE-23 (1985) 25-34. doi:10.1109/TGRS.1985.289497. 
[15] J. Krupka, A.P. Gregory, O.C. Rochard, R.N. Clarke, B. Riddle, J. Baker-Jarvis, Uncertainty of complex permittivity measurements by split-post dielectric resonator technique, J. Eur. Ceram. Soc. 21 (2001) 2673-2676. doi:10.1016/S0955-2219(01)00343-0.

[16] K. Berkowitz, O. Idolor, M. Pankow, L. Grace, Combined effects of impact damage and moisture exposure on composite radome dielectric properties, in: Comb. Eff. Impact Damage Moisture Expo. Compos. Radome Dielectr. Prop., 2018: p. 10.

[17] J. Mijović, H. Zhang, Molecular Dynamics Simulation Study of Motions and Interactions of Water in a Polymer Network, J. Phys. Chem. B. 108 (2004) 2557-2563. doi:10.1021/jp036181j. 